\title{
Una aproximación a los desórdenes hereditarios del ciclo de la urea en el hombre.
}

Revisión

Ivonne Martín-Hernández.

Laboratorio de Errores Innatos del Metabolismo. Centro Nacional de Genética Médica. La Habana, Cuba.

\section{RESUMEN.}

Objetivos. Este trabajo ofrece una revisión teórica y actualizada sobre los defectos hereditarios del ciclo de la urea, dirigida no sólo al personal médico especializado en este tema, sino también a otros especialistas interesados en el estudio de los errores innatos del metabolismo.

Fuentes de extracción. Artículos actualizados y libros de texto relacionados con el tema. Consulta en Internet.

Resultados. El ciclo de la urea es un mecanismo fundamental en los mamíferos para excretar el nitrógeno que no es utilizado. Este involucra cinco enzimas: Carbamil fosfato sintetasa, Ornitina transcarbamilasa, Arginosuccinato sintetasa, Arginosuccinato liasa y Arginasa. La N-acetyl glutamato sintetasa es otra enzima la cual es esencial para el adecuado funcionamiento del ciclo. El malfuncionamiento de alguna de estas enzimas resulta en hiperamonemia. Las manifestaciones clínicas incluyen anorexia, irritabilidad, letargia, desorientación, vómitos, somnolencia, edema cerebral, coma y muerte. Las manifestaciones clínicas pueden aparecer en el período neonatal y ser fatales o aparecer un tiempo después con grados variables de severidad. El diagnóstico de estos desórdenes consiste en la medición de diferentes metabolitos en sangre y orina y en el análisis de las actividades enzimáticas. El tratamiento requiere restricción nutricional de proteínas, administración de aminoácidos específicos y la activación de otras vías de síntesis y excreción del nitrógeno desechado.

Conclusiones. Debido a lo poco frecuente de estos desórdenes, las áreas de salud y los médicos tienen relativamente poca experiencia en el manejo de los mismos. Por esta razón la sospecha del diagnóstico tempranamente y la pronta transferencia a un centro especializado es vital.

(Rev Biomed 2005; 16:193-206)

Palabras clave: Ciclo de la urea, hiperamonemia, diagnóstico, tratamiento.

\section{SUMMARY.}

An approach to hereditary disorders of urea cycle in the man.

Objectives. This work offers a theoretical and

Solicitud de sobretiros: Lic. Ivonne Martín-Hernández, Centro Nacional de Genética Médica, Centro colaborador de la OMS para el desarrollo de enfoques genéticos en la promoción de salud. Calle 146 No. 3102 esq. ave 31. Marianao C.P. 11600. La Habana, Cuba. Correo electrónico: ivonne.martin@infomed.sld.cu

Recibido el 17/Enero/2005. Aceptado para publicación el 23/Mayo/2005.

Este artículo está disponible en http://www.uady.mx/sitios/biomedic/revbiomed/pdf/rb051637.pdf

Vol. 16/No. 3/Julio-Septiembre, 2005 


\section{Martín-Hernández.}

updated review on hereditary disorders of the urea cycle, directed not only to medical professionals in this topic, but also to other specialists interested in the study of inborn errors of metabolism.

Sources of information. Updated articles and books related to the theme. Internet search.

Results. The urea cycle is a fundamental mechanism in mammals in which nitrogen that is not used can be excreted. It involves five enzymes: Carbamylphosphate synthetase, Ornithine transcarbamilase, Arginosuccinate synthetase, Arginosuccinate liase, and Arginase. N-acetyl glutamate synthetase is another enzyme which is essential for the proper operation of the cycle. Malfunction of any of these enzymes results in hyperammonemia. Clinical expression includes anorexia, irritability, lethargy, vomiting, somnolence, desorientation, cerebral edema, coma, and death. The clinical manifestations may appear in the neonatal period and can be fatal, or they may appear any time thereafter with varying degrees of severity. Fundamentally, the diagnosis of these disorders consists of the measurement of different metabolites in blood and urine, and the analysis of enzyme activity. The treatment requires nutritional restriction of proteins, administration of specific amino acids and activation of other pathways of waste nitrogen synthesis and excretion.

Conclusions. Professionals in health facilities and other physicians have relatively little experience in dealing with these disorders, because these disorders are rare. Hence, early suspicion of the diagnosis of a urea cycle disorder, and prompt referral to specialized center is vital. (Rev Biomed 2005; 16:193-206)

Key words: Urea cycle, hiperammonemia, diagnosis, treatment.

\section{INTRODUCCIÓN.}

El hombre excreta el exceso de nitrógeno resultante de la degradación metabólica de los aminoácidos y otros compuestos nitrogenados mediante el ciclo de la urea. Este ciclo es una vía metabólica de cinco pasos, la cual resulta en la conversión de dos moléculas de amonio y una de bicarbonato en urea. Mediante el ciclo de la urea se realiza además la biosíntesis y degradación de arginina, el cual es un aminoácido semi-esencial que modula el desarrollo de la enfermedad cardiovascular ateroesclerótica, mejora la función inmune de los pacientes sanos o enfermos, estimula la curación de heridas y modula la carcinogénesis y el crecimiento tumoral (1-5).

La urea como producto final se sintetiza en el hígado, luego se secreta a la sangre y finalmente es captada por los riñones para excretarla en la orina (2).

El ciclo de la urea fue el primer ciclo metabólico conocido, elucidado en líneas generales en el año 1932 por Hans Krebs y Kart Henseleit. Sus reacciones particulares fueron descritas en detalle más tarde por Sarah Ratner y Philip Cohen (2).

La correcta biosíntesis de urea es necesaria, de forma que la deficiencia de una de las enzimas de la ureagénesis o el fallo de transporte de sus metabolitos implica la síntesis inadecuada de urea y la acumulación de amonio en todas las células del organismo.

Los defectos del ciclo de la urea (DCU) están entre los errores congénitos del metabolismo más comunes y se estima una prevalencia global de las deficiencias de las cinco enzimas del ciclo de 1 : 30,000 recién nacidos (4). Se presentan en todas las edades, de diagnóstico usualmente fácil, pero frecuentemente pasadas por alto, y a menudo tratables (5).

\section{BIOQUIIMICA Y REGULACIÓN DEL CICLO.}

El ciclo de la urea comprende un conjunto de cinco reacciones metabólicas. Figura 1

Carbamoil fosfato sintetasa I (CPS I) es la primera enzima del ciclo de la urea, la cual forma carbamoil fosfato, a partir del amonio y iones bicarbonato con energía proveniente de dos moléculas de ATP. Esta reacción es esencialmente irreversible y es el paso limitante del ciclo de la urea. Esta enzima usa iones magnesio como cofactor y es dependiente de Nacetilglutamato como activador alostérico. Está localizada en la mitocondria del hígado y puede

\section{Revista Biomédica}




\section{Desórdenes hereditarios del ciclo de la urea en el hombre.}

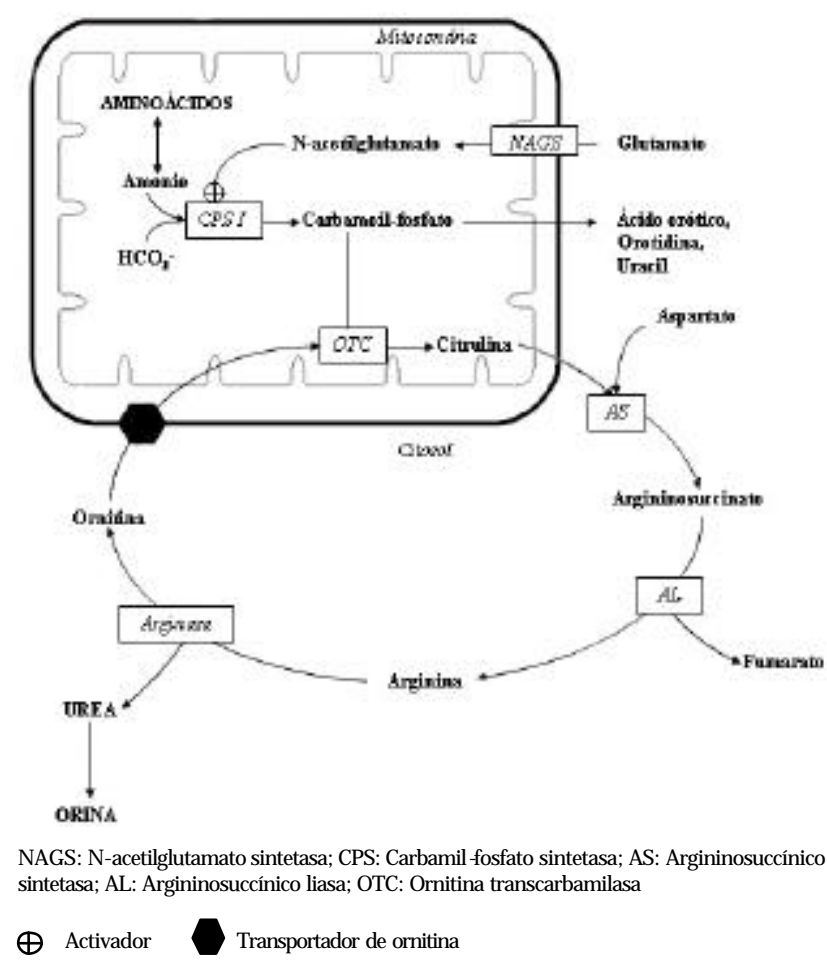

Figura 1.- El ciclo de la urea.

constituir tanto como el $25 \%$ de la proteína de este organelo. El déficit de esta enzima es conocido como hiperamonemia congénita tipo I.

La ornitina transcarbamilasa (OTC) es la segunda enzima del ciclo, transfiriendo el grupo carbamoil del carbamoil fosfato a la ornitina, produciendo citrulina. Esta reacción tiene lugar en la mitocondria, por lo que la ornitina, que se produce en el citosol, debe entrar a la mitocondria a través de un sistema de transporte específico. Igualmente, dado que el ciclo de la urea ocurre en el citosol, la citrulina debe ser exportada de la mitocondria. El déficit de esta enzima es conocido como hiperamonemia congénita tipo II.

El segundo átomo de nitrógeno de la urea se introduce a la tercera reacción del ciclo cuando un grupo ureido de la citrulina se condensa con el grupo amino del aspartato por acción de la argininosuccinato sintetasa $(A S)$. El déficit de esta enzima se denomina citrulinemia.

La vía metabólica prosigue con la enzima arginosuccínico liasa (AL), cuya acción lleva a la producción de fumarato y arginina. El déficit de esta enzima recibe el nombre de acidemia arginosuccínica. El último paso del ciclo de la urea conduce a la ruptura de la arginina en urea y ornitina por acción de la enzima arginasa, conociéndose este déficit como argininemia $(4,6,7)$.

La velocidad de producción de la urea por el hígado está en relación con la concentración de Nacetilglutamato, metabolito sintetizado a partir de glutamato y acetil $\mathrm{CoA}$ por la $\mathrm{N}$-acetil glutamato sintetasa. Cuando aumenta la degradación de aminoácidos generando un exceso de nitrógeno que debe ser excretado, se requiere un aumento en la velocidad de síntesis de la urea. Los incrementos en esta tasa de degradación implican un aumento en la concentración de glutamato a través de las reacciones de transaminación. Esta situación, a su vez, provoca un aumento en la síntesis de $\mathrm{N}$-acetilglutamato, estimulando a la $C P S$ y, por lo tanto, a todo el ciclo de la urea.

Las enzimas restantes del ciclo de la urea están controladas por las concentraciones de sus sustratos. De esta forma, las deficiencias heredadas de las enzimas del ciclo de la urea, excepto la arginasa, no provocan una disminución significativa en la producción de urea (la falta total de cualquier enzima del ciclo de la urea tiene como consecuencia la muerte poco después del nacimiento). Lo que ocurre es que al acumularse el sustrato de la enzima deficiente, aumenta la velocidad de la reacción deficiente hasta niveles normales. La acumulación anómala de sustrato no está, sin embargo, exenta de consecuencia. Las concentraciones de los sustratos se vuelven elevadas a lo largo de todo el ciclo incluyendo al amoníaco, produciéndose una hiperamonemia. Aunque la causa última de la toxicidad del amoníaco no se conoce completamente, una concentración elevada de amoníaco pone mucha presión sobre el sistema eliminador del amoníaco, especialmente en el cerebro. En este sistema de eliminación participan la glutamato deshidrogenasa y la glutamina sintetasa que hace decrecer las reservas de $\alpha$-cetoglutarato y glutamato. El cerebro es muy sensible al agotamiento de estas reservas. El agotamiento del $\alpha$-cetoglutarato disminuye 
I Martín-Hernández.

Cuadro 1

Aspectos bioquímicos de los defectos del ciclo de la urea.

\begin{tabular}{|c|c|c|c|c|c|c|}
\hline Enzima & $\begin{array}{cr}\begin{array}{c}\text { Bases } \\
\text { genéticas }\end{array} & M \\
\end{array}$ & $\begin{array}{l}\text { Metabolitos cara } \\
\text { Sangre }\end{array}$ & $\begin{array}{l}\text { terísticos } \\
\text { Orina }\end{array}$ & $\begin{array}{c}\text { Compar- } \\
\text { timento }\end{array}$ & $\begin{array}{c}\text { Distribución } \\
\text { en tejidos } \\
\end{array}$ & $\begin{array}{c}\text { Confirmación } \\
\text { diagnóstica }\end{array}$ \\
\hline$N A G S$ & $\begin{array}{l}\text { 17q21.31; } 7 \\
\text { exones; } 4 \mathrm{~kb} ; \\
\text { pocas mut. }\end{array}$ & $\begin{array}{l}\mathrm{NH}_{3} \\
\text { Orn, Ala, Gln }\end{array}$ & & $\mathrm{MM}^{*}$ & $\begin{array}{l}\text { Hígado, } \\
\text { intestino, riñón } \\
\text { (trazas), pulmón }\end{array}$ & AE en hígado \\
\hline CPS I & $\begin{array}{l}\text { 2q35; } 38 \\
\text { exones; } 120 \\
\text { kb; pocas mut. }\end{array}$ & $\begin{array}{l}\mathrm{NH}_{3} \\
\text { Gln, Ala, Lys, } \\
\text { Ctr, Arg, Orn }\end{array}$ & rmal) Orotato & $\mathrm{MM}^{*}$ & $\begin{array}{l}\text { Hígado, } \\
\text { intestino, riñón } \\
\text { (trazas) }\end{array}$ & $\begin{array}{l}\mathrm{AE}^{\dagger} \text { en hígado } \\
\text { o mucosa intestinal }\end{array}$ \\
\hline OTC & $\begin{array}{l}\text { Xp 21.1; } 10 \\
\text { exones; } 73 \mathrm{~kb} ; \\
\sim 100 \text { a.g }{ }^{\ddagger}\end{array}$ & $\begin{array}{l}\mathrm{NH}_{3} \\
\text { Gln, Ala, Lys, F } \\
\text { Ctr, Arg, Orn }\end{array}$ & $\begin{array}{l}\text { Orotato } \\
\text { Pirimidinas }\end{array}$ & MM* & $\begin{array}{l}\text { Hígado, } \\
\text { intestino, riñón } \\
\text { (trazas) }\end{array}$ & $\begin{array}{l}\mathrm{AE}^{\dagger} \text { en hígado } \\
\text { o mucosa intestinal; } \\
\text { análisis mutacional; } \\
\text { prueba de alopurinol }\end{array}$ \\
\hline$A S$ & $\begin{array}{l}\text { 9q34; } 16 \\
\text { exones; } 63 \mathrm{~kb} \text {; } \\
\text { 20 mut. }\end{array}$ & $\begin{array}{l}\mathrm{NH}_{3}, \mathrm{Gln}, \text { Lys } \\
\text { Ctr } \\
\text { Arg }\end{array}$ & $\begin{array}{c}\text { Ctr } \\
\text { Orotato }\end{array}$ & Citosol & $\begin{array}{l}\text { Hígado, riñón, } \\
\text { fibroblastos, } \\
\text { cerebro (trazas) }\end{array}$ & $\begin{array}{l}\mathrm{AE}^{\dagger} \text { en hígado y } \\
\text { fibroblastos }\end{array}$ \\
\hline$A L$ & $\begin{array}{l}\text { 7cen-q11.2; } 16 \\
\text { exones; } 35 \mathrm{~kb} \\
\sim 20 \text { mut }\end{array}$ & $\begin{array}{l}\mathrm{NH}_{3} \quad \mathrm{AA} \\
\mathrm{AAS}^{\S}+\text { Anhídri } \\
\text { Ctr, Gln, Lys } \\
\text { Arg }\end{array}$ & $\begin{array}{l}\S+\text { Anhídridos } \\
\text { los Orotato }\end{array}$ & Citosol & $\begin{array}{l}\text { Hígado, riñón, } \\
\text { fibroblastos, } \\
\text { cerebro }\end{array}$ & $\begin{array}{l}\mathrm{AE}^{\dagger} \text { en hígado, } \\
\text { eritrocitos y } \\
\text { fibroblastos }\end{array}$ \\
\hline Arginasa & $\begin{array}{l}6 q 23 ; 8 \text { exones; } \\
11.5 \mathrm{~kb} \\
\sim 20 \mathrm{mut}\end{array}$ & $\begin{array}{l}\mathrm{NH}_{3} \\
\mathrm{Arg} \\
\mathrm{CG}^{\|}\end{array}$ & $\begin{array}{l}\text { Orotato } \\
\text { Arg } \\
\text { Cys, Lys, Orn } \\
\text { CG } \\
\text { Pirimidinas }\end{array}$ & Citosol & $\begin{array}{l}\text { Hígado, } \\
\text { eritrocitos, riñón, } \\
\text { cerebro, (trazas) }\end{array}$ & $\begin{array}{l}\mathrm{AE}^{\dagger} \text { en hígado y } \\
\text { eritrocitos }\end{array}$ \\
\hline
\end{tabular}

* matriz mitocondrial † actividad enzimática $\ddagger$ alteraciones génicas

$\S$ Ácido argininsuccínico ^ compuestos guanidino

la velocidad del ciclo de Krebs, generador de energía, mientras que el glutamato es un neurotransmisor como un precursor del $\gamma$-aminobutirato, otro neurotransmisor $(1,2,8,9)$.

\section{PATRÓN DE HERENCIA Y LOCUS CROMOSÓMICO.}

La herencia de todas las enzimas del ciclo de la urea es autosómica recesiva, salvo la ornitina transcarbamilasa que se hereda ligada al cromosoma $\mathrm{X}$. En este caso se puede predecir el fenotipo de los varones portadores del déficit enzimático; en cambio, esto es imposible en las mujeres debido a la inactivación del cromosoma X. Actualmente se han clonado los genes que codifican cada una de las enzimas del ciclo $(6,10-20)$. Cuadro 1.

\section{HIPERAMONEMIA: DENOMINADOR COMÚN.}

El defecto congénito de una de las enzimas del ciclo de la urea (especialmente CPSyOTC) constituye la causa principal de hiperamonemia grave. Sin embargo, se producen hiperamonemias importantes por defectos de transporte de metabolitos intermediarios del ciclo como: lisinuria con intolerancia a proteínas y síndrome de hiperornitinemiahomocitrulinuria-hiperamonemia $(\mathrm{HHH})$. También se encuentran otras múltiples causas asociadas con hiperamonemia listadas en el cuadro 2 (21-24).

Los altos niveles de amonio en sangre y tejidos resultan en toxicidad para el sistema nervioso central. Las hiperamonemias leves o moderadas pueden acompañarse de anorexia, vómitos, fallo de medro, mareos, somnolencia, obnubilación, ataxia,

\section{Revista Biomédica}




\section{Desórdenes hereditarios del ciclo de la urea en el hombre.}

Cuadro 2

Causas de hiperamonemias.

Hiperamonemias secundarias a otros desórdenes metabólicos hereditarios.

Acidemias orgánicas

Alteraciones del metabolismo de los aminoácidos ramificados

Defectos del metabolismo de la cobalamina

Deficiencias de biotinidasa y holocarboxilasa sintetasa

Deficiencia de piruvato carboxilasa

Deficiencia de piruvato deshidrogenasa

Aciduria piroglutámica

Deficiencia de la beta-oxidación de los ácidos grasos

Hiperamonemias no hereditarias

Hiperamonemia neonatal transiente

Síndrome de Reye

Administración de agentes químicos como: valproato, hipoglicina y ácido 4-pentenoico

Suministro de arginina insuficiente (malnutrición)

Derivaciones hepáticas (malformaciones, cirrosis) Insuficiencia hepática (infección, intoxicación)

irritabilidad y espasticidad. Elevaciones superiores pueden asociarse con convulsiones, letargia, apnea o coma y hasta la muerte en caso de que el tratamiento no sea próximo o efectivo. En pacientes que han estado por períodos prolongados en coma hiperamonémico es posible encontrar atrofia cerebral, ventriculomegalia y retraso mental $(4,21-27)$.

Se considera hiperamonemia significativa valores plasmáticos mayores de $150 \mu \mathrm{mol} / \mathrm{L}$ durante el período neonatal y mayores de $80 \mu \mathrm{mol} / \mathrm{L}$ posteriormente, aunque esta consideración dependerá de los valores de referencia de cada laboratorio (usualmente se consideran los valores normales menores de $50 \mu \mathrm{mol} / \mathrm{L})(21,23)$. Amonemias sobre $1000 \mu \mathrm{g} / \mathrm{dL}(\sim 588 \mu \mathrm{mol} / \mathrm{L})$ representan extraordinaria gravedad porque el amonio cruza por difusión la barrera hematoencefálica, alcanzando valores de hasta el doble de su valor plasmático. El amonio intracerebral es removido formando glutamina por la enzima glutamina sintetasa que se localiza en los astrocitos y que no es inducida por hiperamonemia $(4,28)$. El edema cerebral es uno de los signos clínicos más importante en hiperamonemia y se debería al efecto osmótico de la glutamina en los astrocitos (4, 29). El amonio inhibe la transmisión sináptica en concentraciones sobre $500 \mu \mathrm{mol} / \mathrm{L}$ y en defectos del ciclo de la urea se han reportado valores de amonio cerebral de hasta $5000 \mu \mathrm{mol} / \mathrm{L}$. El amonio estimula la liberación de serotonina alterando la neurotransmisión serotoninérgica. El neurotransmisor serotonina, derivado del triptófano, se acumula al aumentar el nivel de amonio, explicando la anorexia, trastornos del sueño y la insensibilidad al dolor que presentan estos pacientes $(4,30)$.

Las crisis de hiperamonemia pueden cursar con elevación moderada de las transaminasas, sobre todo las formas de presentación tardías (21).

Desafortunadamente los episodios frecuentes de hiperamonemia recurrentes tienen un efecto negativo con desenlace neurológico, constituyendo un problema común debido a las dificultades con la dieta a largo plazo y al manejo farmacológico (31).

\section{CLÍNICA.}

La presentación clínica en pacientes con deficiencias de CPS, $O T, A L$ y $A S$ es virtualmente idéntica, pero con gran variabilidad dentro y entre cada deficiencia. Las manifestaciones clínicas pueden aparecer en el período neonatal y ser fatales en cualquier momento después, con grados variables de severidad. La similitud de la presentación clínica está relacionada con la hiperamonemia, la cual es común a todas y la variabilidad puede estar relacionada con factores génicos así como con las consecuencias metabólicas de varias deficiencias enzimáticas $(6,25)$.

Es conveniente dividir cada una de las deficiencias en dos grupos clínicos: uno con manifestaciones presentes en el período neonatal y otro en que se presenta en un tiempo posterior. En el período neonatal aparecen los síntomas rápidamente progresivos, usualmente en el segundo día de vida. Comienzan los vómitos, rechazo alimentario, letargia o irritabilidad, taquipnea y una rápida progresión al coma y convulsiones. El deterioro es muy rápido con cambio en el tono y pérdida de los reflejos, inestabilidad vasomotora e hipotermia y apnea. Sin 


\section{Martín-Hernández.}

tratamiento los pacientes fallecen, generalmente por complicaciones como hemorragia cerebral o pulmonar, las que enmascaran el defecto metabólico que ocasiona la descompensación. Los que sobreviven esta crisis invariablemente tendrán secuelas neurológicas.

En infantes los síntomas son menos agudos e incluyen anorexia, vómitos, letargia, retardo en el crecimiento y desarrollo. Pueden presentar ataxia y alteraciones conductuales como autoagresión e hiperactividad. Puede existir hepatomegalia, lo que lleva a plantear diagnósticos como hepatitis o alergias alimentarias.

En adolescentes y adultos los síntomas más frecuentes son neurológicos crónicos y psiquiátricos, con problemas de conducta, episodios de desorientación, letargia, psicosis, encefalopatías recurrente asociadas con altas ingestas de proteínas, catabolismo o estrés. En los casos en que ocurra encefalopatía aguda la recuperación puede ser total o pueden quedar con nivel fluctuante de conciencia y signos neurológicos focales como hemiplegia o ceguera cortical. Sin tratamiento, el deterioro es progresivo presentando coma y eventualmente muerte por edema cerebral. Entre los episodios, los pacientes pueden quedar con secuelas neurológicas de magnitud muy variable. Algunos evitan en forma voluntaria la ingesta de proteínas. Generalmente se plantea el diagnóstico diferencial con varias enfermedades, como encefalitis, síndrome de Reye, intoxicación o enfermedad psiquiátrica $(4-6,32,33)$.

Específicamente para la deficiencia de OTC (DOTC) existen tres fenotipos reconocidos: infantes varones severamente afectados, los cuales desarrollan hiperamonemia profunda rápidamente después del nacimiento; varones mayores presentando episodios recurrentes de hiperamonemia y mujeres heterocigóticas afectadas con hiperamonemia recurrente (34).

Los pacientes con aciduria arginosuccínica presentan retardo mental, hepatomegalia severa en las formas tempranas y la mitad de ellos tienen el pelo con tricorrexis nodosa. Los pacientes con déficit de arginasa presentan una displejia espástica, siendo catalogados como parálisis cerebral $(3,28)$. Pueden presentar también ataxia que empeora con los cuadros infecciosos intercurrentes. Sin embargo, los síntomas neurológicos son lentamente progresivos (4, 35-39).

\section{DIAGNÓSTICO.}

Para lograr un diagnóstico correcto de las deficiencias del ciclo de la urea se deberá realizar un examen clínico cuidadoso, acompañado de un estudio de laboratorio apropiado que incluya la determinación de metabolitos en fluidos biológicos. Se deberán realizar determinaciones fundamentales en plasma de amonio, el equilibrio ácido-base, glicemia, lactato, piruvato, aminoácidos, y en orina de aminoácidos y ácido orótico. El análisis de aminoácidos se realiza por cromatografía en columna cuantitativa automatizada debido a que las cromatografias en capa delgada y en papel no son lo suficientemente confiables para este propósito.

Deberán indicarse estudios complementarios para la cuantificación de carnitina total y libre en sangre, y de ácidos orgánicos en orina por cromatografía gaseosa acoplada a espectrometría de masas (4-6, 21, 40-42).

Las neuroimágenes pueden ser de extraordinaria utilidad, pudiendo encontrar un extenso y generalizado edema cerebral durante las crisis encefalopáticas. También se pueden ver las alteraciones de la señal localizada y subsecuente atrofia cerebral en estas áreas (4).

El diagnóstico diferencial de las hiperamonemias se basa en el estudio de metabolitos en plasma y orina del paciente antes de la instauración del tratamiento (21). Figura 2.

El diagnóstico de la deficiencia de $C P S$, se realiza inicialmente por exclusión, sin embargo, debido a que tal diagnóstico implica una vida comprometida a una dieta artificial y molesta medicación, se hace apropiada la medición de la actividad enzimática en hígado obtenida por biopsia percutánea, sin complicaciones en niños después que se establece control metabólico. También se utiliza la medición de la actividad de esta enzima en mucosa intestinal. Estos estudios enzimáticos se deben hacer durante la vida o inmediatamente después de la muerte porque los hallazgos

\section{Revista Biomédica}


Desórdenes hereditarios del ciclo de la urea en el hombre.

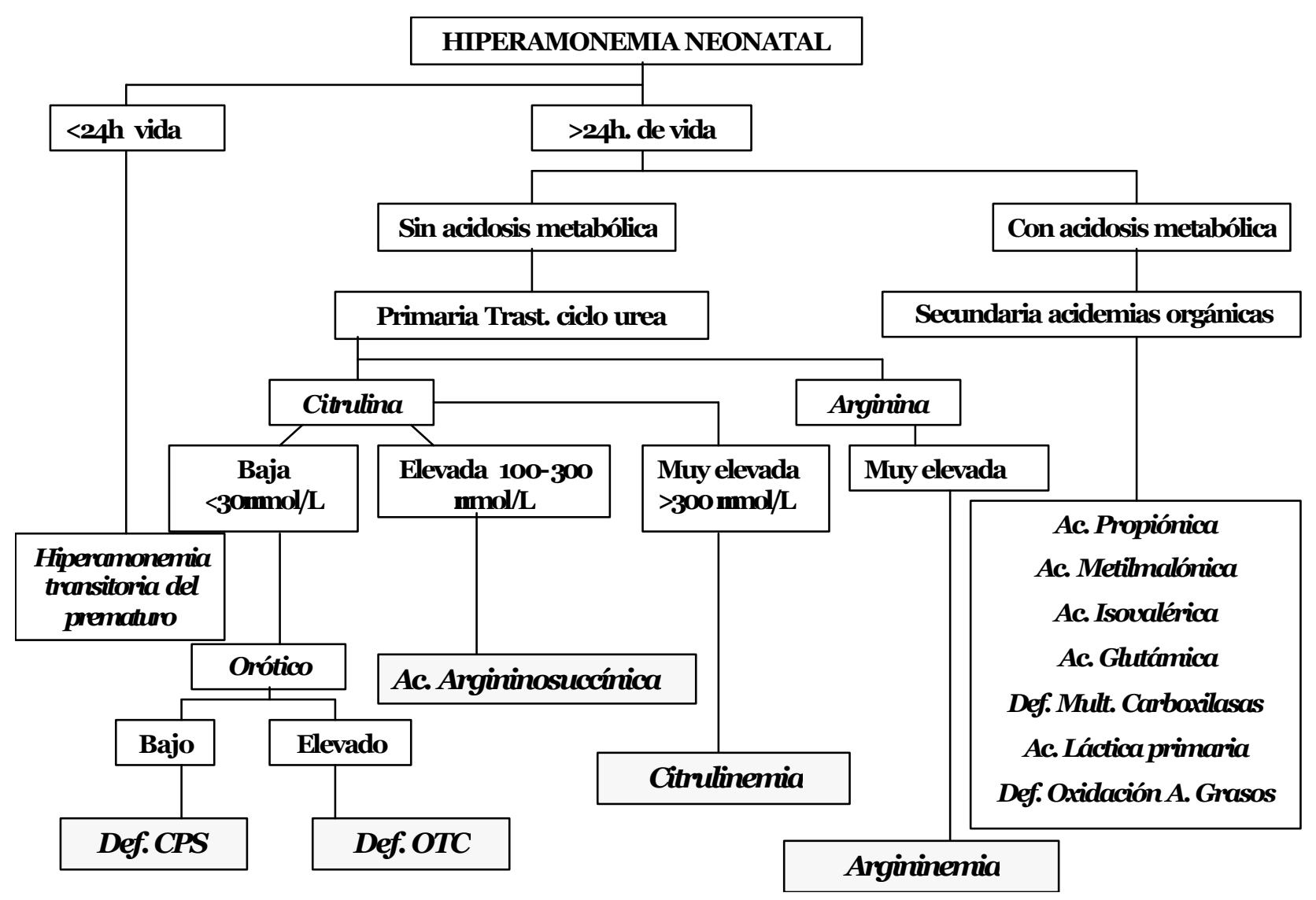

Figura 2.- Diagnóstico diferencial de las hiperamonemias.

postmortem son completamente inespecíficos al inactivarse rápidamente esta enzima mitocondrial. Usualmente se encuentran los niveles de amonio 1020 veces mayores que el rango de referencia y el ácido orótico en valores normales. Los cambios de aminoácidos en sangre no son específicos pero sí nos permiten una diferenciación entre esta deficiencia y los otros desórdenes del ciclo $(6,21,37-40)$.

En pacientes con deficiencia de CPS o OTC, la citrulina se encuentra en concentraciones bajas en plasma debido a que la misma es un producto de ambas enzimas. Para ambos déficits enzimáticos, otras anormalidades en los niveles de aminoácidos en plasma incluyen incremento de glutamina y disminución de los niveles de ornitina y arginina $(6,25,40-42)$. A pesar de que no pueden distinguirse uno del otro por análisis de aminoácidos, ambas deficiencias pueden diferenciarse porque en la deficiencia de OTC existen niveles altos en orina de ácido orótico y pirimidinas incluyendo uracil, uridina y pseudouridina (43).
El análisis del árbol genealógico familiar puede ser útil. En el caso de que no se mida el ácido orótico, los análisis mutacionales pueden ser útiles en definir la enzima afectada. La prueba de alopurinol puede ser útil en el caso de que el análisis mutacional sea infructuoso. Para la detección de heterocigotos de OTC (si el ácido orótico es normal) es importante la realización de la prueba de alopurinol y una sobrecarga oral de proteínas, aunque la primera sea más sensible, específica y segura. Estas pruebas no están exentas de riesgos y están sujeras a falsos positivos o negativos. En ocasiones, la aciduria orótica inducida por el alopurinol puede ser mínima en casos moderados y es suceptible a falsos positivos en pacientes con defectos en la cadena respiratoria (44). Además, la determinación in vivo de la ureagénesis puede usarse como un complemento efectivo de los métodos bioquímicos y moleculares en el diagnóstico de la DOTC en mujeres síntomáticas o asintomáticas y varones con aparición tardía de esta deficiencia. Para

Vol. 16/No. 3/Julio-Septiembre, 2005 


\section{Martín-Hernández.}

ello uno de los métodos utilizados es la introducción de glutamina marcada con $\mathrm{N}^{15}$ en su grupo amino como fuente de nitrógeno, correlacionando la relación $\mathrm{N}^{15}$ urea/ $\mathrm{N}^{15}$ glutamina con la severidad clínica (45).

La medición de la actividad enzimática de laOTC puede ser ambigua en la mujer, debido a que el hígado es un mosaico de hepatocitos y se han encontrado diferencias en la actividad de la OTC en muestras de biopsias del mismo hígado. También se ha reportado que la actividad de esta enzima en vivo puede ser normal en mujeres sintomáticas. Puede ser útil la medición de la actividad de la enzima OTC en mucosa intestinal, aunque la experiencia es limitada (46).

En un paciente sintomático a citrulinemia, la determinación de amonio en plasma es la primera prueba de laboratorio a realizar. El siguiente paso a seguir será la determinación de aminoácidos en plasma, encontrándose niveles de citrulina entre 1000 y 5000 $\mu \mathrm{M}$ (valores normales 10 a $20 \mu \mathrm{M}$ ). En estos pacientes se encuentran niveles elevados de ácido orótico en orina.

La acidemia argininosuccínica se caracteriza por la presencia de altas concentraciones de argininosuccinato y sus anhidridos, los cuales no aparecen normalmente en el plasma. Se debe tener en cuenta en el análisis de infantes con coma hiperamonémico, que el argininosuccinato puede no identificarse porque coeluye con los aminoácidos presentes normalmente, a menos que el tiempo de retención del mismo sea conocido. Los niveles de citrulina en plasma aparecen moderadamente incrementados (100 a $300 \mu \mathrm{M})$ al igual que de glutamina, alanina y lisina $(6,23,41)$.

El diagnóstico de argininemia puede realizarse sobre las bases de una moderada concentración de arginina en suero (o tan altos como $1500 \mu \mathrm{M}$ ), test de sobrecerga y la deficiencia de la arginasa en eritrocitos como diagnóstico bioquímico definitivo. La excreción de arginina en orina usualmente no está masivamente incrementada debido a la inducción de isoenzimas, sin embargo, el patrón de aminoácidos en orina de la argininemia se parece al obtenido en cistinuria-lisinuria con concentraciones aumentadas de cistina, lisina y ornitina, las cuales se excretan en orina debido a la inhibición competitiva de la reabsorción de aminoácidos dibásicos en los túbulos proximales renales (6). Una variedad de compuestos guanidino, como $\mathrm{N}$-acetilarginina, ácido $\alpha$-ceto $\delta$ guanidinovalérico, ácido arginínico y homoarginina, se encuentran en suero, orina y fluido cerebroespinal (47, 48). Otra característica encontrada es incremento en la excreción de ácido orótico, aunque los niveles de amonio en plasma pueden estar medianamente elevados o ser normales. Otras pirimidinas como uracil y uridina se excretan en la orina en cantidades mayores que lo normal. En pacientes afectados, la deficiencia de la actividad enzimática se demuestra en hígado, eritrocitos, leucocitos y saliva, pero no en fibroblastos o riñón. Ha habido reportes de análisis inmunoquímicos de argininemia $(6,23,49)$.

\section{DIAGNÓSTICO PRENATAL.}

Los cinco defectos de la ureagénesis pueden diagnosticarse prenatalmente. Las técnicas empleadas varían ampliamente e incluyen medición de metabolitos anormales en líquido amniótico, análisis de ácido desoxiribunucleico (ADN) de las vellosidades coriónicas o amniocitos y actividad enzimática en muestras de biopsia de hígado fetal (6).

Para fetos con riesgo de deficiencia de CPS y cuando se dispone del ADN de un miembro afectado de la familia, se emplea el estudio de ligamientos utilizando los polimorfismos en la longitud de los fragmentos de restricción (RFLP). En caso de que no existan RFLP informativos, se puede realizar el diagnóstico en biopsias de hígado fetal $(6,23,50)$. Existen cuatro marcadores flanqueadores útiles y el análisis mutacional de una secuencia larga codificable está disponible también (6).

Para fetos con riesgo de deficiencia de $O T C$, el procedimiento de elección es la secuenciación marcada cuando la mutación se ha identificado previamente, o la secuenciación de todos los exones del locus OTC. Sólo cuando las mujeres se han definido como portadoras de la deficiencia de $O T C$, ya sea por análisis de árbol genealógico, prueba de alopurinol o por análisis mutacional, puede emplearse en el diagnóstico prenatal el análisis de RFLP. En caso de no resultar informativo por RFLP, el diagnóstico puede 


\section{Desórdenes hereditarios del ciclo de la urea en el hombre.}

ser realizado por análisis mutacional o biopsia de hígado fetal en fetos masculinos. El papel del diagnóstico prenatal y la terminación del embarazo de fetos femeninos con riesgo es controversial porque éste requiere un estimado de la probabilidad del feto para convertirse en sintomático $(6,23,51)$.

En fetos con riesgo de deficiencia de $A S$ y que tengan ausencia de RFLP informativo es necesaria la medición de la actividad de la enzima en vellosidades coriónicas, aunque este método ofrece el riesgo del bajo nivel de actividad en fetos heterocigóticos. Este problema se supera mediante el empleo de una técnica radiométrica basada en la incorporación de citrulina$\mathrm{C}^{14}$ en fibroblastos derivados de amniocitos para distinguir homocigosis de heterocigosis $(6,23,52)$.

Existen dos técnicas fiables para el diagnóstico prenatal de la deficiencia de $A L$ : medición de actividad enzimática en cultivo de amniocitos y medición de los niveles de ácido argininosuccínico en líquido amniótico. Un número limitado de mutaciones se ha identificado en algunos individuos $(19,53)$.

En fetos con riesgo de deficiencia de arginasa, la medición de actividad enzimática fetal en eritrocitos parece ser la técnica más satisfactoria a pesar de la disponibilidad de algunos marcadores intragénicos (6, 37).

\section{TRATAMIENTO.}

El coma hiperamonémico $(>300 \mu \mathrm{mol} / \mathrm{L})$ es una emergencia médica en la que debe instaurarse un tratamiento inmediato y agresivo en un intento de prevenir o minimizar un posible daño cerebral irreversible $(21,54)$.

En estos casos se requiere supresión absoluta de proteínas orales o parenterales, reintroduciendo éstas en 48 a 72 horas $(0,25 \mathrm{~g} / \mathrm{kg}$ a $0,5 \mathrm{~g} / \mathrm{kg})$ cuando se haya conseguido estabilizar las concentraciones de amonio plasmático por debajo de $100 \mu \mathrm{mol} / \mathrm{L}$, y valorando la tolerancia de las mismas con controles diarios de amonemia. Se debe administrar liquídos suficientes para mantener una correcta situación hemodinámica, pero teniendo en cuenta la posible existencia de edema cerebral y aportar calorías necesarias por vía parenteral central en forma de solución glucosada (10-15\%) para limitar el catabolismo endógeno y enteral (polímeros de glucosa) con aporte de 150 a $200 \mathrm{kcal} / \mathrm{kg} /$ día (4, 6, 21).

Para disminuir la absorción de amonio desde la luz intestinal se utiliza lactulosa por vía oral o en forma de enemas, junto con la administración de ciertos antibióticos como la neomicina (21).

Mientras no se conozca el diagnóstico etiológico exacto de la hiperamonemia se debe administrar clorhidrato de arginina (L-arginina $\mathrm{HCl}$ ) y carnitina por vía intravenosa a las dosis de $250-500 \mathrm{mg} / \mathrm{kg} /$ día y $22 \mathrm{mg} / \mathrm{kg} /$ día, respectivamente. La arginina facilita la activación del ciclo de la urea a través de la $N A G S$ y $C P S$ I. La carnitina ha demostrado su eficacia en la eliminación de productos tóxicos en forma de acilcarnitinas, en las acidemias orgánicas, en los trastornos de la beta-oxidación de los ácidos grasos, así como en las fases agudas de descompensación de los trastornos del ciclo de la urea. La administración de altas dosis de arginina $\mathrm{HCl}$ puede producir acidosis metabólica hiperclorémica. Un grado ligero de acidosis puede reducir la cantidad de amonio que traspasa la barrera hematoencefalíca en dirección al sistema nervioso central al mantener una baja relación $\mathrm{NH}_{3} /$ $\mathrm{NH}_{4}+(6,21,22,54)$.

Cuando tenemos confirmado el diagnóstico de defecto congénito del ciclo de la urea, la administración de benzoato sódico y/o fenilbutirato sódico en la fase aguda puede contribuir a la más rápida normalización de la amonemia. El benzoato sódico puede administrarse en dosis de 200-500 mg/ $\mathrm{kg} /$ día por sonda nasogástrica o por vía intravenosa. El fenilbutirato sódico puede administrarse a $250 \mathrm{mg} /$ $\mathrm{kg}$ /día en combinación con el benzoato, o de 400$650 \mathrm{mg} / \mathrm{kg} /$ día, como medicación única. La administración de altas dosis de benzoato puede producir irritabilidad, náuseas, vómitos, colapso cardiopulmonar y acidosis metabólica. El fenilbutirato parece tolerarse mejor.

El objetivo del uso de estos compuestos consiste en la excreción de nitrógeno en forma de productos diferentes de la urea (vías alternativas). El benzoato se conjuga con la glicina formando hipurato, excretándose así un mol de nitrógeno por cada mol 


\section{Martín-Hernández.}

de benzoato administrado. El fenilbutirato se combina con la glutamina formando fenilacetilglutamina, consiguiéndose la eliminación de dos mol de nitrógeno por cada mol de fenilbutirato. La excreción de hipurato y fenilacetilglutamina aumenta la pérdida urinaria de potasio pudiendo conducir a una hipopotasemia y alcalosis metabólica $(4,6,21-23,54)$.

Cuando las medidas expuestas hasta el momento no son suficientes para controlar la hiperamonemia, como puede ocurrir en situaciones de sepsis o shock, acudiremos a los métodos de diálisis según las posibilidades de cada centro: hemofiltración, hemodialfiltración, hemodiálisis y diálisis peritoneal (4, 6, 21-23, 54).

Todos los pacientes con trastornos del ciclo de la urea tienen riesgo de descompensación metabólica que puede precipitarse por diversas situaciones de estrés, como un ayuno prolongado, excesiva ingesta proteica, infección, anestesia, cirugía, etc. Por tal razón, los pacientes y/o familiares deben saber cómo actuar ante situaciones de riesgo (como un episodio infeccioso) o sospecha de descompensación, con sus síntomas característicos (21).

El tratamiento crónico para mantener los niveles de amonio cerca de la normalidad (o dentro del rango normal para la edad según los valores establecidos en cada laboratorio), consiste en el establecimiento de un régimen nutricional donde la ingesta de proteínas naturales de valor biológico será entre 0,7 y $1,5 \mathrm{~g} / \mathrm{kg} /$ día (leche humana, fórmulas infantiles). En las variantes más graves puede ser díficil conseguir un buen control metabólico y una nutrición satisfactoria únicamente por restricción de estas proteínas (sobre todo en las deficiencias de CPS y OTC). Algunos pacientes presentan una importante anorexia y no ingieren suficiente cantidad de proteína natural. En ambas situaciones parte de la proteína natural (habitualmente menos del $50 \%$ ) puede sustituirse con fórmulas especiales que contienen sólo aminoácidos esenciales (1,4 - 2,0 g/kg/día) (4, 6, 21, 55). Cuadro 3.

En situación de importante restricción proteíca, algunos aminoácidos no esenciales pueden convertirse en limitantes de la síntesis de productos importantes para el metabolismo intermediario como la carnitina

\section{Revista Biomédica}

\section{Cuadro 3}

Concentraciones plasmáticas recomendadas de aminoácidos.

\begin{tabular}{lc}
\hline Aminoácido & $\mu \mathrm{mol} / \mathrm{L}$ \\
\hline Arginina & $80-150$ \\
Ácido aspártico & $14-50$ \\
Citrulina & $20-30$ \\
Glutamina & $<800$ \\
Glicina & $100-170$ \\
Serina & $100-170$ \\
Treonina & $>80$ \\
Leucina & $>70$ \\
Isoleucina & $>40$ \\
Valina & $>120$ \\
\hline
\end{tabular}

(lisina y metionina) o glutatión (cisteína y glutamato) (21).

La cantidad de proteína tolerada puede aumentar cuando se administran productos que proporcionan vías alternativas para la eliminación de amonio. La cantidad de proteína suministrada debe ser suficiente para permitir un crecimiento correcto debido a que un aporte proteíco bajo conlleva a la pérdida de peso, fallo de medro, hipoalbuminemia, osteopenia, caída del cabello e hiperamonemias inexplicadas $(4,6,21$ 23, 54, 55). Cuadro 4.

Se recomienda un aporte calórico elevado, superior a las necesidades para la edad (hasta un $20 \%$ ), para evitar catabolismo excesivo y favorecer anabolismo. Los hidratos de carbono no deben ser superiores al $60 \%$ del total de calorías para evitar hipertrigliceridemias. Un tratamiento con excesivo aporte de calorías induce a ganancia de peso y/o talla del percentil $50(4,55,56)$.

\section{Cuadro 4}

Rango de proteínas totales y calorías recomendadas para los niños con trastornos congénitos del ciclo de la urea.

\begin{tabular}{ccc}
\hline Edad(años) & $\begin{array}{c}\text { Proteína total } \\
(\mathrm{g} / \mathrm{kg} / \mathrm{dí} a)\end{array}$ & $\begin{array}{c}\text { Calorías } \\
(\mathrm{kcal} / \mathrm{kg} / \mathrm{dí} a)\end{array}$ \\
\hline $0-1$ & $1,2-2,2$ & $20-145$ \\
$1-7$ & $1,0-1,2$ & $100-120$ \\
$7-9$ & $0,7-1,4$ & $80-110$ \\
$>19$ & $0,5-1,0$ & $35-65$ \\
\hline
\end{tabular}




\section{Desórdenes hereditarios del ciclo de la urea en el hombre.}

Todos los pacientes con déficit enzimáticos del ciclo de la urea, excepto el déficit de arginasa, necesitan un suplemento de arginina para mantener los niveles plasmáticos entre 50 y $150 \mu \mathrm{mol} / \mathrm{L}$. En los defectos mitocondriales ( $C P S$ y OTC) las necesidades de arginina están alrededor de 100-150 mg/kg/día, y la arginina puede sustituirse por la citrulina, a unas dosis similares, la cual consigue la eliminación suplementaria de un nitrógeno procedente del aspartato, además de la producción de arginina. Los pacientes con citrulinemia y aciduria arginosuccínica precisan más cantidad de arginina (400-700 mg/kg/día) por la pérdida urinaria importante de ornitina incorporada a la citrulina y argininosuccínico (4, 6, 21-23, 54-56).

En la deficiencia de $N A G S$ puede administrarse $\mathrm{N}$-carbamilglutamato a dosis de $100-300 \mathrm{mg} / \mathrm{kg} / \mathrm{día}$, vigilando la posible aparición del síndrome del "restaurante chino" (21).

Las vitaminas del complejo B (cianocobalamina, niacina, folato, ácido pantoténico y piridoxina) pueden mejorar la síntesis de glicina y la conjugación de glicina y benzoato y de fenilacético con glutamina. En niños sometidos a dietas hipoproteíacas se ha observado una deficiencia de selenio, por lo que se recomienda un suplemento de elementos traza. Suplementar zinc y calcio según recomendaciones establecidas $(4,6$, 21-23, 54-56).

El transplante de hígado ortotópico como una forma de reemplazo enzimático es una terapia médica alternativa para las deficiencias severas del ciclo de la urea, particularmente en pacientes con deficiencias de $C P S$ y $O T C$. Muchos reportes apuntan al éxito de esta terapia proporcionando un control metabólico y la prevención de episodios adicionales de hiperamonemia, preservando la función neurológica. Originalmente el transplante fue evitado entre niños menores de 12 meses de edad debido al riesgo de muerte y morbilidad. Los avances en las técnicas quirúrgicas y el manejo de la inmunosupresión nos permiten hoy en día el uso del transplante en edades tempranas, con tasas de mortalidad similares a las obtenidas en niños mayores. Sin embargo cuando el transplante se lleva a cabo posteriormente, el compromiso neurológico ya existe y éste no se corrige
$(57,58)$.

Se ha utilizado también el transplante de hepatocitos aislados a partir de hígados donados en infantes afectados severamente con los DCU, y en mujeres heterocigóticas para la deficiencia de $O T C$, en los cuales un pequeño incremento de la actividad de la $O T C$ previene las crisis hiperamonémicas recurrentes y preserva el neurodesarrollo (59).

La frecuencia del seguimiento de los pacientes con DCU dependerá de la comprensión de la dieta por parte de los padres, de la evolución del niño, presencia de infecciones y síntomas sugerentes de hiperamonemia. Anualmente se deberá realizar la densitometría ósea, resonancia magnética nuclear y electroencefalograma si hay convulsiones (4).

\section{CONCLUSIONES.}

Hasta el momento la población cubana no ha sido investigada para las DCU por lo que la verdadera incidencia de estos desórdenes se desconoce. Debido a lo raro de estos desórdenes las áreas de salud y los médicos tienen relativamente poca experiencia en el manejo de los mismos. Por esta razón la sospecha del diagnóstico tempranamente y la pronta transferencia a un centro especializado es vital.

\section{REFERENCIAS.}

1.- Voet Cohen PP. The ornitine-urea cycle: biosynthesis and regulation of carbamyl phosphate synthetase I and ornitine transcarbamilase. Curr Top Cell Regul 1981; 18: 119.

2.- Voet D, Voet JG. Bioquímica. 1a. ed. Barcelona: Ediciones Omega; 1992.p. 725-78.

3.- Tong BC, Balbul A. Cellular and physiological effects of arginine. Mini Rev Med Chem 2004; 4: 823-32.

4.- Cornejo V, Raimann E. Errores innatos del metabolismo de los aminoácidos. En: Colombo M, Cornejo V, Raimann E, editores. Errores innatos del metabolismo del niño. $2^{\mathrm{a}}$ ed. Santiago de Chile: Editorial universitaria SA; 2003. p. 71138.

5.- Zschocke J, Hoffmann G. Metabolic pathways and their disorders. En: Zschocke J, Hoffmann G, editores. Vademecum

Vol. 16/No. 3/Julio-Septiembre, 2005 


\section{Martín-Hernández.}

metabolicum. Manual of metabolic Pedriatrics. $2^{\mathrm{a}}$ ed Stuttgart: Milupa GmbH \& Co. KG; 1999. p. 35-56.

6.- Brusilow S, Horwich A. Urea cycle enzymes. En: Scriver Ch, Beaudet A, Sly W, Valle D, editores. The metabolic and molecular bases of inherited disease. $8^{\text {th }}$ ed. New York: McGraw-Hill Inc; 2001.vol. 2: 1909-64.

7.- Rainmann E, Cornejo V, Mellibosky D, López ME, Godoy X, Colombo M. Citrulinemia. Rev Chil Pediatr 1994; 65: 2158.

8.- Meijer AJ, Lamers WH, Chamuleau R. Nitrogen metabolism and ornitine cycle function. Physiol Rev 1990; 70: 701-48.

9.- Morris SM. Regulation of enzymes of urea and arginine síntesis. Annu Rev Nutr 1992; 12: 81-101.

10.- Caldovic L, Morizono H, Panglao MG, Gallegos R, Yu X, Shi D, et al. Cloning and expression of the human Nacetylglutamate synthase gene. Biochem Biophys Res Commun 2002; 209: 581-6.

11.- Summar ML, Hall LD, Eeds AM, Hutcheson HB, Kuo AN, Willis AS, et al. Characterization of the structure and polimorphisms in the human carbamyl phosphate synthetase I gene. Gene 2003; 311: 51-7.

12.- Sheehy L, Lynch MG. Mutations and polymorphisms in the human ornithine transcarbamylase gene. 2002; 19: 93 107.

13.- Haberle J, Pauili S, Linneebank M, Kleijer WJ, Bakker HD, Wanders RJ, et al. Structure of the human argininosuccinate synthetase gene and the improved system for molecular diagnostics in patients with classical and mild citullinemia. Hum Genet 2002; 110: 327-33.

14.- Kobayashi K, Kakinoki H, Fukushige T, Shaheen N, Terazono H, Saheki T. Nature and frecuency of mutations in the argininosuccinate synthetase gene that cause classical citullinemia. Hum Genet 1995; 96: 454-63.

15.- Online Mendelian Inheritance in Man: $\mathrm{N}$-acetyl glutamate synthetase, 608300. Available from: URL: http// www.ncbi.nlm.nih.gov/OMIM.

16.- Online Mendelian Inheritance in Man: Carbamoyl phosphate synthetase, 608307. Available from: URL: http// www.ncbi.nlm.nih.gov/OMIM.

17.- Online Mendelian Inheritance in Man: Ornithine transcarbamilase, 300461. Available from: URL: http// www.ncbi.nlm.nih.gov/OMIM.

18.- Online Mendelian Inheritance in Man: Arginosuccinate synthetase, 603470. Available from: URL: http// www.ncbi.nlm.nih.gov/OMIM.

19.- Online Mendelian Inheritance in Man: Arginosuccinate lyase, 608310. Available from: URL: http// www.ncbi.nlm.nih.gov/OMIM.

20.- Online Mendelian Inheritance in Man: Arginase, 608313. Available from: URL: http//www.ncbi.nlm.nih.gov/OMIM.

21.- Pintos G, Briones MP, Marchante C, Sanjurjo P,. Protocolo para el diagnóstico, tratamiento y seguimiento de los trastornos del ciclo de la urea. An Esp Pediatr 1997; 89: 1-8.

22.- Leonard JV. Urea cycle disorders. En:Fernández J, Saudubray JM, Van der Berge G, editores. Inborn metabolic diseases. Berlín: Springer-Verlag; 1995. p. 167-176.

23.- Wilcox WR, Cederbaum SD. Amino acid metabolism. En: Rimoin E, Connor JM, Pyeritz BR, editores.Emery and Rimoin`s. Principles and practice of medical genetics. $4 \mathrm{a}$ ed. New york: Churchill Livingtone; 2002. Vol 2: 2405-40.

24.- Walker V. Hipoglycaemia and hyperamonaemia. En: Clinical biochemistry and the sick child. Clayton BE, Round JM, editores. Oxford: Blackwell Scientific Publications; 1994. p. 87-120.

25.- Mathias RS, Kostiner D, Packman S. Hyperammonemia in urea cycle disorders: Role of the nephrologist. Am J Kidney Dis 2001;37: 1069-80.

26.- Batshaw ML. Inborn errors of urea synthesis. Ann Neurol 1994; 35: 133-41.

27.- Batshaw ML: Hyperammonemia. Curr Probl Pediatr 1984; 14: 1-69.

28.- Ratnakumari L, Qureshi IA, Butterworth RF. Regional aminoacid neurotransmitter changes in brains of spf/Y mice with congenital ornithine transcarbamilase deficiency. Metab Brain Dis 1994; 9: 43-52.

29.- Blei A, Olafsson Therrien G, Butterworth RF. Ammonia induced brain edema and intracranial hypertension in rats after portocaval anastomosis. Hepatology 1994; 19: 143744.

\section{Revista Biomédica}




\section{Desórdenes hereditarios del ciclo de la urea en el hombre.}

30.- Butterworth R. Effects of hyperammnonemia on brain function. J Inhit Metab Dis 1998; 21: 6-20.

31.- Uchino T, Endo F, Matsuda I. Neurodevelopmental outcome of long-term therapy of urea cycle disorders in Japan. J Inherited Metab Dis 1998; 21(suppl 1); 151-9.

32.- Nicolaides P, Liebsh D, Leonard J, Surtees R. Neurological outcome of patients with ornithine carbamoyltransferase deficiency. Arch Dis Child 2002, 86:546.

33.- Hudak ML, Jones MD, Brusilow. Differentiation of transient hyperammonemia of the newborn and urea cycle enzyme defects by clinical presentation. J Pedriatr 1985; 107 : 712-9.

34.- Nicolaides P, Liebsch D, Dale N, Leonard J, Surtees R. Neurological outcome of patients with ornithine carbamoyltransferase deficiency. Clin Chem 1999; 45: 995 1001.

35.- Widhalm K, Koch S, Scheibenreiter S. Long-term followup of 12 patients with the late-onset variant of argininosuccinic acid lyase deficiency: no impairment of intellectual and psychomotor development during therapy. Pediatrics 1992; 89 (6 Pt 2): 1182-4.

36.- Cowley DM, Bowling FG, McGill JJ, van Dongen J. Adultonset arginase deficiency. J Inherit Metab Dis 1998; 21: 6778 .

37.- Iyer R, Jenkinson CP, Vockley JG, Kern RM. The human arginases and arginase deficiency. J Inherit Metab Dis 1998; 21(Suppl 1): 86-100.

38.- Picker JD, Puga AC, Levy HL. Arginase deficiency with lethal neonatal expression: evidence for the glutamine hypothesis of cerebral edema. J Pediatr 2003; 142: 349-52.

39.- Scheuerle AE, McVie R, Beaudet AL, Shapira SK. Arginase deficiency presenting as cerebral palsy. Pediatrics 1993;91:995-6.

40.- Batshaw ML, Thomas GH, Brusilow SW. New approaches to the diagnosis and treatment of inborn errors of urea synthesis. Pediatrics 1981; 68:290-7.

41.- Steiner RD, Cederbaum SD. Laboratory evaluation of urea cycle disorders. J Pediatr 2001; 138 (1 Pt 2): 21-29. 96.-

42.- Summar M. Current strategies for the management of neonatal urea cycle disorders. J Pedriat 2001; 130: 30-39.
43.- Webster DR, Simmons HA, Barry DMJ, Becroft DMO. Pyrimidine and purine metabolites in ornithine carbamoyl transferase deficiency. J Inherited Metab Dis 1981; 4 :27-31.

44.- Arranz JA, Riudor E, Rodés M, Roig M, Climent C, Rubio $\mathrm{V}$, et al. Optimization of allopurinol challenge: sample purification, protein intake control, and the use of oritidine response as a discriminative variable improve performance of the test for diagnosing orotidine carbamoyltransferase deficiency. Pediatrics 2003; 111: 1123-4.

45.- Scaglia F, Zheng Q, O'Brien WE, Henry J, Rosenberger $\mathrm{J}$, Reeds $\mathrm{P}$, et al. An integrated approach to the diagnosis and prospective management of partial ornithine transcarbamylase deficiency. Pedriatrics 2002; 109: 150-2.

46.- Nagata N, Endo F, Matsuda I. Ornithine carbamoyltransferase (OTC) in jejunal mucosa, as a reference of the liver OTC. Clin Chim Acta 1983; 134: 155-66.

47.- Marescau B, Qureshi IA, De Deyn P, Letarte J, Ryba R, Lowenthal A. Guanidino compounds in plasma, urine and cerebrospinal fluid of hyperargininemic patients during therapy. Clin Chim Acta 1985; 146: 21-7.

48.- Marescau B, Lowenthal A. Isolation and identification of some guanidino compounds in the urine of patients with hyperargininaemia by liquid chromatography, thin-layer chromatography and gas chromatography spectrometry. J Chromatogr 1981; 224: 185-8.

49.- Naylor EW, Cederbaum SD. Urinary pyrimidine excretion in arginase deficiency. $\mathrm{J}$ Inherit Dis 1981; 4: 207-10.

50.- Finckh U, Kohlschutter A, Schafer H, Sperhake. Prenatal diagnosis of carbamoyl phosphate synthetase I deficiency by identification of a missense mutation in CPS1. Hum Mutat 1998; 12: 206-11.

51.- Fox J, Hack AM, Fenton WA, Golbus MS, et al: Prenatal diagnosis of ornithine transcarbamylase deficiency with use of DNA polymorphisms. N Engl J Med 1986; 315: 1205-8.

52.- Hayakawa M, Kato Y, Takahashi R, Tauchi N. Case of citrullinemia diagnosed by DNA analysis: including prenatal genetic diagnosis from amniocytes of next pregnancy. Pediatr Int 2003; 45: 196-8.

53.- Haberle J, Koch HG. Genetic approach to prenatal diagnosis in urea cycle defects. Prenat Diagn 2004; 24 : 37883 .

Vol. 16/No. 3/Julio-Septiembre, 2005 


\section{Martín-Hernández.}

54.- Wilcken B. Problems in the management of urea cycle disorders. Mol Genet Metab 2004; 81 (Suppl 1): 86-91.

55.- Leonard J. The nutricional management of urea cycle disorders. J Pediatr 2001; 138, 40-45.

56.- Berry GT, Steiner RD: Long-term management of patients with urea cycle disorders. J Pediatr 2001; 138(1 Pt 2): 56-62.

57.- McBride KL, Miller G, Carter S, Karpen S, Goss J, Lee B. Developmental Outcomes With Early Orthotopic Liver Transplantation for Infants With Neonatal-Onset Urea Cycle Defects and a Female Patient With Late-Onset Ornithine Transcarbamylase Deficiency. Pediatrics 2004; 114: 523-6.

58.- Lee B, Goss J. Long-term correction of urea cycle disorders. J Pediatr. 2001; 138:62 -71.

59.- Horslen SP, McCowan TC, Goertzen TC, Warkentin PI, Bo Cai H, Strom SC, et al. Isolated hepatocyte transplantation in an infant with a severe urea cycle disorder. Pediatrics 2003;111:1262-7.

\section{Revista Biomédica}

